\title{
Topology-Preserving Thinning in 2-D Pseudomanifolds
}

\author{
Nicolas Passat ${ }^{1}$, Michel Couprie ${ }^{2}$, Lö̈c Mazo ${ }^{1,2}$, and Gilles Bertrand ${ }^{2}$ \\ ${ }^{1}$ Université de Strasbourg, LSIIT, UMR CNRS 7005, Strasbourg, France \\ ${ }^{2}$ Université Paris-Est, Laboratoire d'Informatique Gaspard-Monge, \\ Équipe A3SI, ESIEE Paris, France \\ \{passat, loic.mazo\}@unistra.fr, \{m.couprie,g.bertrand\}@esiee.fr
}

\begin{abstract}
Preserving topological properties of objects during thinning procedures is an important issue in the field of image analysis. In the case of 2-D digital images (i.e. images defined on $\mathbb{Z}^{2}$ ) such procedures are usually based on the notion of simple point. By opposition to the case of spaces of higher dimensions (i.e. $\mathbb{Z}^{n}, n \geq 3$ ), it was proved in the 80's that the exclusive use of simple points in $\mathbb{Z}^{2}$ was indeed sufficient to develop thinning procedures providing an output that is minimal with respect to the topological characteristics of the object. Based on the recently introduced notion of minimal simple set (generalising the notion of simple point), we establish new properties related to topology-preserving thinning in 2-D spaces which extend, in particular, this classical result to more general spaces (the 2-D pseudomanifolds) and objects (the 2-D cubical complexes).
\end{abstract}

Keywords: Topology preservation, simple points, simple sets, cubical complexes, collapse, confluence, pseudomanifolds.

\section{Introduction}

Topological properties are fundamental in many applications of image analysis, in particular in cases where the retrieval and/or the preservation of topology of real complex structures is required. In this context, numerous methods have been developed to process discrete 2-D and 3-D binary images, essentially to perform skeletonisation, homotopic thinning, or segmentation.

Such methods are generally based on the notion of simple point [8]. Intuitively, a point (or pixel) of a discrete object $X$ is said to be simple if it can be removed from $X$ without altering its topology.

Let us consider an object $X$, i.e. a set of points in $\mathbb{Z}^{n}$, and a subset $Y$ of $X$ called constraint set. A very common topology-preserving thinning scheme [4] consists of repeating the following steps until stability:

- choose (according to some given priority function) a point $x$ in $X \backslash Y$ that is simple for $X$;

- remove $x$ from $X$.

The result of such a procedure, called homotopic skeleton of $X$ constrained by $Y$, is a subset $Z$ of $X$, which $(i)$ is topologically equivalent to $X$, (ii) includes $Y$ and (iii) has no simple point outside of $Y$. We show an illustration in Fig. 1, notice in particular that the constraint set is useful to preserve some geometrical characteristics of the object. 


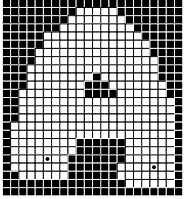

(a)

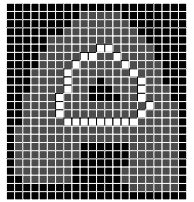

(b)

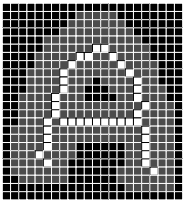

(c)

Fig. 1. (a) An object $X$ (in white) and a subset $Y$ of $X$ (two pixels marked by black dots). (b) A homotopic skeleton of $X$ (empty constraint set). (c) A homotopic skeleton of $X$ constrained by $Y$.

The following question is fundamental with regard to the behaviour of sequential thinning procedures:

(1) Is $Z$ always a minimal result, in the sense that it does not strictly include a subset $Z^{\prime}$ having the same properties $(i),(i i)$ and (iii)?

If we consider the 3-D case, the answer to this question is no. For example, if $X$ is a cuboid and $Y=\emptyset$, then, depending on the order of the point removals, the result $Z$ of the above procedure might not be composed of a single point. As pointed out recently [11], there exist various kinds of configurations in which a 3-D topology-preserving thinning algorithm can be "blocked" before reaching a minimal result.

In the discrete plane $\mathbb{Z}^{2}$, question (1) was answered positively by Ronse in the 80's, after a partial answer was given in the early 70's by Rosenfeld. In 1970, in the same article where he introduced the notion of simple point [15], Rosenfeld proved that any finite subset of $\mathbb{Z}^{2}$ that is connected and has no holes, could be reduced to a single point by iterative removal of simple points, in any order. In 1986, Ronse introduced the notion of strong deletability in $\mathbb{Z}^{2}[14]$. It is, to the best of our knowledge, the first attempt to generalise the notion of simple points to a more general notion of simple sets.

According to Def. 2.5 of [14], and skipping formal details, a subset $S$ of $X \subseteq \mathbb{Z}^{2}$ is strongly deletable from $X$ if $(i)$ each connected component of $X$ includes exactly one connected component of $X \backslash S$, and (ii) each connected component of $\bar{X} \cup S$ includes exactly one connected component of $\bar{X}$, where $\bar{X}$ denotes the complementary of $X$.

In the same article, Ronse proposed several results related to strongly deletable sets, which can be summarised as follows.

Theorem 1 (From [14], Lem. 3.1, 3.2, Prop. 3.3). Let $X \subseteq \mathbb{Z}^{2}$. Let $S \subseteq X$. If $S$ is strongly deletable from $X$, then:

(i) there exists $x \in S$ such that $x$ is a simple point for $X$;

(ii) for all $x \in S$ such that $x$ is a simple point for $X, S \backslash\{x\}$ is strongly deletable for $X \backslash\{x\}$.

Consequently, if $Y \subseteq X \subseteq \mathbb{Z}^{2}$ and $Y$ is topologically equivalent to $X$ (more precisely, if $X \backslash Y$ is strongly deletable from $X$ ), then $Y$ may be obtained from $X$ by iterative removal of simple points, in any arbitrary order.

To summarise, question (1) received a positive answer in $\mathbb{Z}^{2}$ and a negative one in $\mathbb{Z}^{3}$ (and also for higher dimensions). Still, there are spaces for which this question remained 
open until now: the case of two-dimensional structures in $n$-dimensional spaces, $n \geq 3$. Such structures are often used in practice, e.g. to represent thin objects or (parts of) boundary of objects in 3-D image analysis and in finite element modelling.

The main outcome of this article is a theorem (Th. 26) that states a property analogous to Th. 1, holding in a large family of 2-D digital spaces, namely the pseudomanifolds.

This study is developed in the framework of cubical complexes [9], in which we can retrieve and generalise the concepts of digital topology in $\mathbb{Z}^{n}$. The definition of simple sets that we use here is based on the operation of collapse, a topology-preserving transformation known in algebraic topology.

The proof of Th. 26 is based on a property of collapse, that we call a confluence property (Th.21), which is introduced and proved in this article.

Th. 26 is also closely related to the notion of minimal simple set [11]13], as we derive it using the following property: if $X$ is a strict subset of a pseudomanifold, then any minimal simple subset of $X$ is a simple point (Prop. 25).

Thanks to a correspondence between the notion of minimal simple set used here and the one of simple point [7], we retrieve as particular cases of Th.26 the results of Rosenfeld and Ronse discussed before. However, the techniques of proof used in this article are essentially different from the ones used by these authors, and the generalisation of their results is not trivial.

This article is self-contained, however some of the proofs cannot be included due to space limitation (they can however be found in the following research report [12]).

\section{Background Notions}

In this section, we provide basic definitions and properties related to the notions of cubical complexes, collapse and simple sets (the last two ones enabling to modify a complex without altering its topology), see also [9]13].

\subsection{Cubical Complexes}

If $T$ is a subset of $S$, we write $T \subseteq S$. Let $\mathbb{Z}$ be the set of integers. Let $k, \ell \in \mathbb{Z}$, we denote by $[k, \ell]$ the set $\{i \in \mathbb{Z} \mid k \leq i \leq \ell\}$.

We consider the families of sets $\mathbb{F}_{0}^{1}, \mathbb{F}_{1}^{1}$, such that $\mathbb{F}_{0}^{1}=\{\{a\} \mid a \in \mathbb{Z}\}$ and $\mathbb{F}_{1}^{1}=$ $\{\{a, a+1\} \mid a \in \mathbb{Z}\}$. A subset $f$ of $\mathbb{Z}^{n}(n \geq 2)$ that is the Cartesian product of $m$ elements of $\mathbb{F}_{1}^{1}$ and $n-m$ elements of $\mathbb{F}_{0}^{1}$ is called a face or an $m$-face of $\mathbb{Z}^{n}, m$ is the dimension of $f$, we write $\operatorname{dim}(f)=m$ (see Fig. $2(\mathrm{a}, \mathrm{b})$ ).

Let $n \geq 2$, we denote by $\mathbb{F}^{n}$ the set composed of all faces of $\mathbb{Z}^{n}$.

An $m$-face of $\mathbb{Z}^{n}$ is called a point if $m=0$, a (unit) edge if $m=1$, a (unit) square if $m=2$.

Let $f$ be a face in $\mathbb{F}^{n}$. We set $\hat{f}=\left\{g \in \mathbb{F}^{n} \mid g \subseteq f\right\}$. Any $g \in \hat{f}$ is a face of $f$ (or of $\hat{f}$ ).

If $X$ is a set of faces of $\mathbb{F}^{n}$, we write $X^{-}=\bigcup_{f \in X} \hat{f}$, and we say that $X^{-}$is the closure of $X$.

A set $X$ of faces of $\mathbb{F}^{n}$ is a cell or an $m$-cell if there exists an $m$-face $f \in X$, such that $X=\hat{f}$. The boundary of a cell $\hat{f}$ is the set $\hat{f}^{*}=\hat{f} \backslash\{f\}$ (see Fig. 2). 
A finite set $X$ of faces of $\mathbb{F}^{n}$ is a complex (in $\mathbb{F}^{n}$ ) if for any $f \in X$, we have $\hat{f} \subseteq X$.

Let $S, X$ be two sets of faces of $\mathbb{F}^{n}$. If $X$ is a complex and $X \subseteq S$, we write $X \leq S$. Furthermore, if $S$ is also a complex, then we say that $X$ is a subcomplex of $S$.

Let $X \subseteq \mathbb{F}^{n}$. A face $f \in X$ is a facet of $X$ if there is no $g \in X$ such that $f \in \hat{g}^{*}$, in other words, if $f$ is maximal for inclusion. A facet of $X$ that is an $m$-face is also called an $m$-facet of $X$. We denote by $X^{+}$the set composed of all facets of $X$ (see Fig. 3).

If $X$ is a complex, observe that in general, $X^{+}$is not a complex, and that $\left(X^{+}\right)^{-}=X$. More generally, for any subset $Y$ of $\mathbb{F}^{n},\left(Y^{+}\right)^{-}=Y^{-}$.

Let $X \subseteq \mathbb{F}^{n}, X \neq \emptyset$. The dimension of $X$ is the number $\operatorname{dim}(X)=\max \{\operatorname{dim}(f) \mid$ $f \in X$, and we set $\operatorname{dim}(\emptyset)=-1$. We say that $X$ is pure if for each $f \in X^{+}$, we have $\operatorname{dim}(f)=\operatorname{dim}(X)$. Let $m$ be an integer. We say that $X$ is an $m$-complex if $X$ is a complex and $\operatorname{dim}(X)=m$. If $X$ is an $m$-complex with $m \leq 1$, then we also say that $X$ is a graph (see [5]).

Let $Y \leq X \leq \mathbb{F}^{n}$. If $Y^{+} \subseteq X^{+}$, we say that $Y$ is a principal subcomplex of $X$ and we write $Y \sqsubseteq X$ (see Fig. 4).

Let $X \subseteq \mathbb{F}^{n}$. A sequence $\pi=\left\langle f_{i}\right\rangle_{i=0}^{\ell}\left(\ell \geq 0\right.$ ) of faces in $X$ is a path in $X$ (from $f_{0}$ to $f_{\ell}$ ) if for each $i \in[0, \ell-1]$, either $f_{i}$ is a face of $f_{i+1}$ or $f_{i+1}$ is a face of $f_{i}$; the integer $\ell$ is the length of $\pi$. The path $\pi$ is said to be closed whenever $f_{0}=f_{\ell}$, it is a trivial path whenever $\ell=0$.

Let $X \subseteq \mathbb{F}^{n}$. A path in $X$ made of 0 - and 1-faces is called a 1-path. A 1-path from a 0 -face $x$ to a 0 -face $y$ (with possibly $x=y$ ), is said to be elementary if its 1 -faces are all distinct. A non-trivial elementary closed path is called a cycle.

Let $X \subseteq \mathbb{F}^{n}$. We say that $X$ is connected if, for any pair of faces $(f, g)$ in $X$, there is a path in $X$ from $f$ to $g$. It is easily shown that, if $X$ is a complex, then $X$ is connected if and only if there exists an elementary path from $x$ to $y$ in $X$ whenever $x$ and $y$ are 0 -faces in $X$.

Let $X \subseteq \mathbb{F}^{n}$, and let $Y$ be a non-empty subset of $X$, we say that $Y$ is a connected component of $X$ if $Y$ is connected and if $Y$ is maximal for these two properties (i.e., if we have $Z=Y$ whenever $Y \subseteq Z \subseteq X$ and $Z$ is connected). We will sometimes write component as a shortcut for connected component. The number of components of $X$ is denoted by $|C(X)|$. Notice that $|C(\emptyset)|=0$.

\subsection{Collapse and Simple Sets}

Let $X$ be a complex in $\mathbb{F}^{n}$ and let $f \in X$. If there exists a face $g \in \hat{f}^{*}$ such that $f$ is the only face of $X$ that strictly includes $g$, then $g$ is said to be free for $X$, and the pair $(f, g)$ is said to be a free pair for $X$. Notice that, if $(f, g)$ is a free pair for $X$, then we have necessarily $f \in X^{+}$and $\operatorname{dim}(g)=\operatorname{dim}(f)-1$.

Let $X$ be a complex, and let $(f, g)$ be a free pair for $X$. Let $m=\operatorname{dim}(f)$. The complex $X \backslash\{f, g\}$ is an elementary collapse of $X$, or an elementary m-collapse of $X$.

Let $X, Y$ be two complexes. We say that $X$ collapses onto $Y$, and we write $X \searrow Y$, if $Y=X$ or if there exists a collapse sequence from $X$ to $Y$, i.e., a sequence of complexes $\left\langle X_{i}\right\rangle_{i=0}^{\ell}(\ell \geq 1)$ such that $X_{0}=X, X_{\ell}=Y$, and $X_{i}$ is an elementary collapse of $X_{i-1}$, for each $i \in[1, \ell]$ (see Fig. 5). Let $J=\left\langle\left(f_{i}, g_{i}\right)\right\rangle_{i=1}^{\ell}$ be the sequence of pairs of faces of $X$ such that $X_{i}=X_{i-1} \backslash\left\{f_{i}, g_{i}\right\}$, for any $i \in[1, \ell]$. We also call the sequence $J$ a collapse 
sequence (from $X$ to $Y$ ). If $X$ collapses onto $Y$ and $Y$ is a complex made of a single point, we say that $X$ is collapsible.

Let $Y, X \subseteq \mathbb{F}^{n}$. We say that $X$ is an extension of $Y[1]$ if $Y \subseteq X$ and each connected component of $X$ includes exactly one connected component of $Y$.

Proposition 2 (proved in [12]). Let $Y \leq X \leq \mathbb{F}^{n}$. If $X \searrow Y$, then $X$ is an extension of $Y$. In consequence, collapse preserves the number of connected components.

Let $X \leq \mathbb{F}^{n}$, the complex that is the closure of the set of all free faces for $X$, is called the boundary of $X$, and is denoted by $B d(X)$. We denote by $B d_{1}(X)$ the complex that is the closure of the set of all free 1-faces for $X$ (see Fig. 6). Of course, we have $B d_{1}(X) \leq$ $\operatorname{Bd}(X)$.

Proposition 3 (proved in [12]). Let $Y \leq X \leq \mathbb{F}^{n}$, let $\alpha$ be a set of facets of $X$ that are not in $Y$, i.e., $\alpha \subseteq X^{+} \backslash Y$. If $B d\left(\alpha^{-}\right) \subseteq Y$, then $X$ does not collapse onto $Y$.

Proposition 4 (proved in [12]). Let $Z \leq X \leq \mathbb{F}^{n}$ be two complexes such that $X \searrow Z$. Let $J=\left\langle\left(f_{i}, g_{i}\right)\right\rangle_{i=1}^{\ell}$ be a collapse sequence from $X$ to $Z$. Suppose that there exists $Y \leq X$ such that $Z \leq Y$ and for any $i \in[1, \ell]$, either $\left\{f_{i}, g_{i}\right\} \subseteq Y$ or $\left\{f_{i}, g_{i}\right\} \subseteq X \backslash Y$. Then, $X \searrow Y$ and $Y \searrow Z$.

Let $J=\left\langle\left(f_{i}, g_{i}\right)\right\rangle_{i=1}^{\ell}$ be a collapse sequence. This collapse sequence is said to be $d e$ creasing if for any $i \in[1, \ell-1]$, we have $\operatorname{dim}\left(f_{i}\right) \geq \operatorname{dim}\left(f_{i+1}\right)$.

Proposition 5 ([16]). Let $Y \leq X \leq \mathbb{F}^{n}$. If $X$ collapses onto $Y$, then there exists a decreasing collapse sequence from $X$ to $Y$.

Let $X, Y$ be two complexes. Let $Z$ be such that $X \cap Y \leq Z \leq Y$, and let $f, g \in Z \backslash X$. The pair $(f, g)$ is a free pair for $X \cup Z$ if and only if $(f, g)$ is a free pair for $Z$. Thus, by induction, we have the following property.

Proposition 6 ([2]). Let $X, Y \leq \mathbb{F}^{n}$. The complex $X \cup Y$ collapses onto $X$ if and only if the complex $Y$ collapses onto $X \cap Y$.

The operation of detachment allows us to remove a subcomplex from a complex while guaranteeing that the result is still a complex (see Fig.7).

Definition 7 ([2]). Let $Y \leq X \leq \mathbb{F}^{n}$. We set $X \otimes Y=(X \backslash Y)^{-}$. The set $X \otimes Y$ is a complex that is called the detachment of $Y$ from $X$.

Intuitively a cell $\hat{f}$ or a subcomplex $Y$ of a complex $X$ is simple if its removal from $X$ "does not modify the topology of $X$ ". Let us now recall a definition of simplicity [2] based on the collapse operation, which can be seen as a discrete counterpart of the one given by Kong [7].

Definition 8 ([2]). Let $Y \leq X \leq \mathbb{F}^{n}$. We say that $Y$ is simple for $X$ if $X$ collapses onto $X \otimes Y$. 


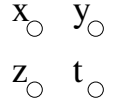

(a)

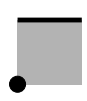

(b)

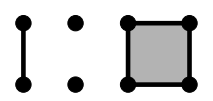

(c) (d) (e)

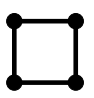

(f)

Fig. 2. (a) Four points of $\mathbb{Z}^{2}: x=(0,1) ; y=(1,1) ; z=(0,0) ; t=(1,0)$. (b) A representation of the set of faces $\left\{f_{0}, f_{1}, f_{2}\right\}$ in $\mathbb{F}^{2}$, where $f_{0}=\{z\}$ ( 0 -face), $f_{1}=\{x, y\}$ (1-face), and $f_{2}=\{x, y, z, t\}$ (2-face). (c) A 1-cell $\hat{c}$. (d) A 2-cell $\hat{d}$. (e) The boundary $\hat{c}^{*}$ of $\hat{c}$. (f) The boundary $\hat{d}^{*}$ of $\hat{d}$.

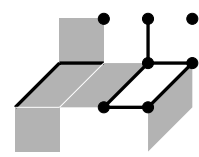

(a)

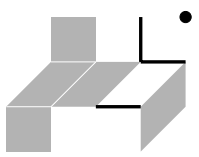

(b)

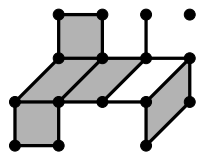

(c)

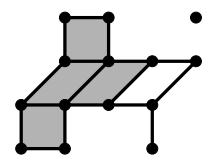

(d)

Fig. 3. (a) A set $X$ of 0-, 1- and 2-faces in $\mathbb{F}^{3}$, which is not a complex. (b) The set $X^{+}$, composed of the facets of $X$. (c) The set $X^{-}$, i.e. the closure of $X$, which is a complex. (d) A subcomplex of $X^{-}$.

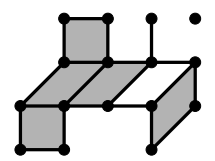

(a)

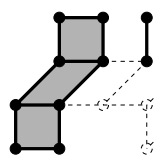

(b)

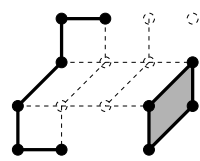

(c)

Fig. 4. (a) A complex $X$. (b) A subset $Y$ of $X$, which is a principal subcomplex of $X$ (i.e., $Y \sqsubseteq X$ ). (c) A subset $Z$ of $X$, which is a subcomplex of $X$ but not a principal subcomplex of $X$.

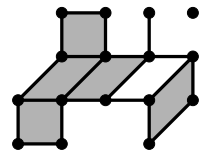

(a)

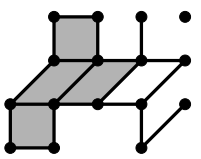

(b)

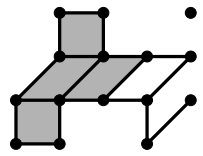

(c)

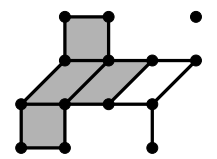

(d)

Fig. 5. (a) A complex $X$. (d) A subcomplex $Y$ of $X$. (a,b,c,d) A collapse sequence from $X$ to $Y$.

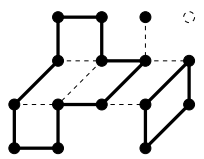

(a)

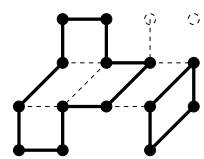

(b)

Fig. 6. (a) $B d(X)$, where $X$ is the complex of Fig.5 a). (b) $B d_{1}(X)$.

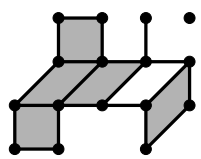

(a)

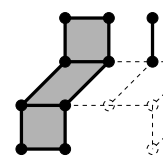

(b)

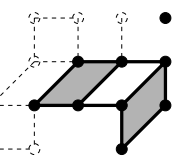

(c)

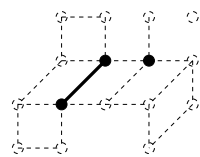

(d)

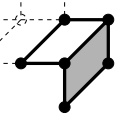

(e)

Fig. 7. (a) A complex $X$. (b) A subcomplex $Y$ of $X$ that is simple for $X$. (c) The detachment of $Y$ from $X$. (d) The attachment of $Y$ to $X$. (e) A subcomplex $Z$ of $X$ that is not simple for $X$. 
If $\hat{f}$ is a simple cell, we will also say that $f$ is simple.

The notion of attachment, as introduced by Kong [6.7], leads to a local characterisation of simple sets (Prop.9).

Let $Y \leq X \leq \mathbb{F}^{n}$. The attachment of $Y$ for $X$ is the complex defined by $\operatorname{Att}(Y, X)=$ $Y \cap(X \ominus Y)$ (see Fig. 7). Remark that any facet $f$ of $X$ such that $\operatorname{Att}(\hat{f}, X) \neq \hat{f}^{*}$ includes a free face for $X$.

Proposition 9 ([2]). Let $Y \leq X \leq \mathbb{F}^{n}$. The complex $Y$ is simple for $X$ if and only if $Y$ collapses onto $\operatorname{Att}(Y, X)$.

Remark 10. If $Y=\emptyset$, or if $Y \leq X$ contains no facet of $X$, then $Y$ is obviously $a$ simple set for $X$, as we have $X \odot Y=X$. More generally, it can be proved [13] that the detachment of a subcomplex $Y$ from $X$ is equal to the detachment of the maximal principal subcomplex $Z$ of $X$ included in $Y$. Without loss of generality, the study of the simple sets $Y$ of a complex $X$ can then be restricted to those verifying $Y \sqsubseteq X$ and $Y \neq \emptyset$. From now on, we will always implicitly consider that a simple set verifies these hypotheses.

\section{Confluence Properties in Cubical Complexes}

Consider three complexes $A, B, C$. If $A$ collapses onto $C$ and $A$ collapses onto $B$, then we know that $A, B$ and $C$ "have the same topology". If furthermore we have $C \leq B \leq A$, it is tempting to conjecture that $B$ collapses onto $C$. We call this a confluence property. For example, this property implies that any complex in $\mathbb{F}^{2}$ obtained by a collapse sequence from a full rectangle indeed collapses onto a point.

Quite surprisingly, such a property does not hold in $\mathbb{F}^{3}$ (and more generally in $\mathbb{F}^{n}, n \geq$ 3). A classical counter-example to this assertion is Bing's house ([3], see also [11]). A realisation of Bing's house as a 2-complex can be obtained by collapse from a full cuboid, and has no free face: it is thus a counter-example for the above conjecture, with $A$ : a cuboid, $B$ : Bing's house, and $C$ : a point in $B$.

As we will show in this article, in the two-dimensional discrete plane $\mathbb{F}^{2}$ and more generally in the class of discrete spaces called pseudomanifolds, a confluence property indeed holds (Th.21).

In this section, we establish confluence properties that are essentially 1-dimensional, a step for proving the more general confluence properties of Section 5 .

A tree is a graph that is collapsible. It may be easily proved that a graph is a tree if and only if it is connected and does not contain any cycle (see [5]).

Let $X \leq \mathbb{F}^{n}$ be a complex. The set of all $i$-faces of $X$, with $i \in[0, n]$, is denoted by $F_{i}(X)$. We denote by $\left|F_{i}(X)\right|$ the number of $i$-faces of $X, i \in[0, n]$. The Euler characteristic of $X$, written $\chi(X)$, is defined by $\chi(X)=\sum_{i=0}^{n}(-1)^{i}\left|F_{i}(X)\right|$. The Euler characteristic is a well-known topological invariant; in particular, it can be easily seen that collapse preserves it.

Let $X, Y \leq \mathbb{F}^{n}$. A fundamental and well-known property of the Euler characteristic, deriving from the so-called inclusion-exclusion principle in set theory, is the following: $\chi(X \cup Y)=\chi(X)+\chi(Y)-\chi(X \cap Y)$.

The following property generalises a classical characterisation of trees: a graph $X$ is a tree if and only if $X$ is connected and $\chi(X)=1$. 
Proposition 11 (proved in [12]). Let $X, Y$ be such that $Y \leq X \leq \mathbb{F}^{n}$, and $\operatorname{dim}(X \backslash Y) \leq 1$. Then, $X$ collapses onto $Y$ if and only if $X$ is an extension of $Y$ and $\chi(Y)=\chi(X)$.

From Props.2111 and the fact that collapse preserves the Euler characteristic, we derive straightforwardly the following two propositions.

Proposition 12. Let $A, B, C$ be such that $C \leq B \leq A \leq \mathbb{F}^{n}$ and such that $\operatorname{dim}(B \backslash C) \leq 1$. If $A$ collapses onto $C$ and $A$ collapses onto $B$, then $B$ collapses onto $C$.

Proposition 13. Let $A, B, C$ be such that $C \leq B \leq A \leq \mathbb{F}^{n}$ and such that $\operatorname{dim}(A \backslash B) \leq 1$. If $A$ collapses onto $C$ and $B$ collapses onto $C$, then $A$ collapses onto $B$.

\section{Pseudomanifolds}

Intuitively, a (2-D) manifold [10] is a 2-D (finite or infinite) space which is locally "like" the 2-D Euclidean space (spheres and tori are, for instance, manifolds).

The notion of (2-D) pseudomanifold is less restrictive since it authorises several pieces of surface to be adjacent in a singular point (as two cones sharing the same apex, for instance). Note that any manifold is a pseudomanifold, but the converse is not true. Some examples of pseudomanifolds are provided in Fig. 8 .

In the framework of cubical complexes, a 2-D pseudomanifold can be defined as follows. We denote by $\mathbb{F}_{2}^{n}$ the set composed of all $m$-faces of $\mathbb{Z}^{n}$, with $m \in[0,2]$. We say that $\pi$ is a 2-path (in $X$ ) if $\pi$ is a path in $X$ composed of 1- and 2-faces.

Definition 14. Let $M \subseteq \mathbb{F}_{2}^{n}$ be such that $\operatorname{dim}(M)=2$. We say that $M$ is a (2-D) pseudomanifold if the following four conditions hold:

(i) for any $f \in M$, we have $\hat{f} \subseteq M$;

(ii) $M$ is pure;

(iii) for any pair of 2-faces $(f, g)$ in $M$, there is a 2-path in $M$ from $f$ to $g$;

(iv) any 1-face of $M$ is included in exactly two 2-faces of $M$.

Notice that, in particular, $\mathbb{F}_{2}^{2}=\mathbb{F}^{2}$ (namely the discrete plane) is a pseudomanifold. Notice also that, if $M$ is a finite pseudomanifold, then $M$ is a pure 2-complex that cannot be collapsed, since $M$ has no free face by definition.

In the sequel, we focus on complexes that are strict subsets of a pseudomanifold.

Proposition 15 (proved in [12]). Let $M \subseteq \mathbb{F}_{2}^{n}$ be a pseudomanifold, and let $X \leq M$. Then, $B d\left(B d_{1}(X)\right)=B d(B d(X))=\emptyset$.

Proposition 16 (proved in [12]). Let $M \subseteq \mathbb{F}_{2}^{n}$ be a pseudomanifold, let $B \leq M$ such that $\operatorname{dim}(B)=2$ and $B \neq M$, let $f$ be a 2 -face of $B$, and let $g$ be a 2 -face in $M \backslash B$. If $\pi$ is a 2-path from $f$ to $g$ in $M$, then $\pi$ necessarily contains a 1 -face of $B d(B)$.

Prop.17ffollows easily from Prop.16,

Proposition 17. Let $M \subseteq \mathbb{F}_{2}^{n}$ be a pseudomanifold, let $B \leq M$. If $\operatorname{dim}(B)=2$ and $B \neq M$, then there exists at least one pair $(f, g)$ that is free for $B$, with $\operatorname{dim}(f)=2$. 


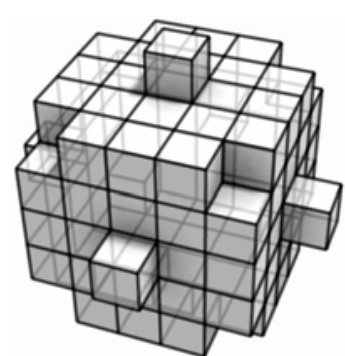

(a)

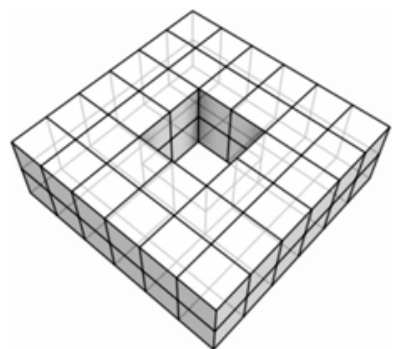

(b)

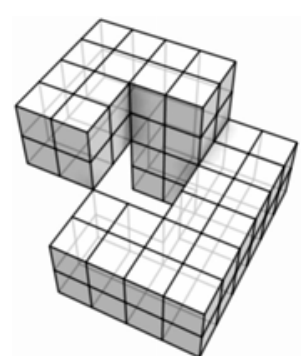

(c)

Fig. 8. 2-D pseudomanifolds. (a) A topological sphere. (b) A topological torus. (c) A pinched torus. (a) and (b) are (pseudo)manifolds, (c) is a pseudomanifold but not a manifold.

\section{Confluence Properties in Pseudomanifolds}

Our goal in this section is to establish confluence properties, similar to Props. 12 and [13, in the case where $A, B$ and $C$ are complexes that are subsets of a pseudomanifold.

It is tempting to try to generalise Prop. 11 to this case, for confluence properties would immediately follow from such a result. But in fact, the backward implication of Prop.11does not hold in the general case (that is, when $\operatorname{dim}(X \backslash Y)$ is not constrained), even if $X$ and $Y$ are complexes that are subsets of a pseudomanifold.

A counter-example is given by $M$ : a pinched torus (see Fig. 8 (c)), $A \leq M$ : a topological disk (e.g., a square and all the faces included in it), $X=M \otimes A$, and $Y=B d(X)=$ $X \cap A$ (a topological circle). It is easily checked that $\chi(M)=\chi(X \cup A)=1, \chi(A)=1$ and $\chi(Y)=0$, and since $\chi(X \cup A)=\chi(X)+\chi(A)-\chi(X \cap A)$ we deduce $\chi(X)=0=\chi(Y)$. We have also $Y \leq X$ and $|C(Y)|=|C(X)|=1$, thus $X$ is an extension of $Y$. However, by construction, $X$ has no free face outside $Y$, thus $X$ does not collapse onto $Y$.

A similar counter-example could be built from a sphere $M$, which is a manifold, and a ring $A$ (a closed ribbon that is a pure 2-complex). In this case $X$ is made of two topological discs and $X \cap A$ is made of two topological circles. We have $\chi(M)=\chi(X)=$ 2 and $\chi(A)=\chi(X \cap A)=0$.

Nevertheless, we have the following property.

Proposition 18 (proved in [12]). Let $M \subseteq \mathbb{F}_{2}^{n}$ be a pseudomanifold, and let $X \leq M$, $X \neq M$. The complex $X$ is collapsible if and only if $|C(X)|=\chi(X)=1$.

Proposition 19 (Downstream confluence). Let $M \subseteq \mathbb{F}_{2}^{n}$ be a pseudomanifold, and let $C \leq B \leq A \leq M$. If $A$ collapses onto $C$ and $A$ collapses onto $B$, then $B$ collapses onto C.

Proof. If $\left|F_{2}(B)\right|=\left|F_{2}(C)\right|$ then by Prop. 12, $B \searrow C$. Suppose that $\left|F_{2}(B)\right|>\left|F_{2}(C)\right|$. Suppose that the proposition holds for any $B^{\prime}$ instead of $B$, with $\left|F_{2}(C)\right| \leq\left|F_{2}\left(B^{\prime}\right)\right|<$ $\left|F_{2}(B)\right|$. Let $q$ be a 2-face of $B$ not in $C$. Since $A \searrow C$ and by Prop. 5, there exists a sequence of 2-collapse operations starting from $A$ and that removes $q$. Let $S$ be the set of faces removed by this sequence, clearly there exists a 2-path $\pi$ in $S$, from a 1face $p$ that belongs to $B d_{1}(A)$, to $q$. Let $h$ be the 2-face just following $p$ in $\pi$. If $h \in B$ 
then necessarily $p \in B d_{1}(B)$; and if $h \notin B$, we deduce from Prop. 16 that $\pi$ contains at least one 1-face of $B d_{1}(B)$. Since by construction $C$ contains no element of $\pi$, we have $B d_{1}(B)^{+} \nsubseteq C$, thus $B$ has a free pair $(f, g)$ that is not in $C$, with $\operatorname{dim}(f)=2$. Let $B^{\prime}=B \backslash\{f, g\}$. Obviously $A \searrow B^{\prime}$, thus by the recurrence hypothesis $B^{\prime} \searrow C$, hence $B \searrow C$.

Proposition 20 (Upstream confluence). Let $M \subseteq \mathbb{F}_{2}^{n}$ be a pseudomanifold, and let $C \leq B \leq A \leq M$. If $A$ collapses onto $C$ and $B$ collapses onto $C$, then $A$ collapses onto $B$.

Proof. If $\left|F_{2}(A)\right|=0$ then by Prop. 13, $A \searrow B$. Suppose that $\left|F_{2}(A)\right|>0$ and that the proposition holds for any $A^{\prime}$ instead of $A$, with $\left|F_{2}\left(A^{\prime}\right)\right|<\left|F_{2}(A)\right|$. Consider the set $\alpha$ of 1-faces that are free for $A$ and not in $C$, i.e., $\alpha=F_{1}(B d(A) \backslash C)$. If $\alpha=\emptyset$, then the hypothesis $A \searrow C$ implies that $\left|F_{2}(A)\right|=\left|F_{2}(C)\right|=\left|F_{2}(B)\right|$, and the result follows from Prop. 13. We now suppose that $\alpha \neq \emptyset$. By Prop. 15, no face in $B d(A)$ is free for $B d(A)$, hence no face in $\alpha^{-}$is free for $\alpha^{-} \cup C$. Thus, all the faces in $\alpha$ cannot be facets of $B$, for otherwise by Prop. 3, $B$ could not collapse onto $C$. From this, we deduce that there exists a 1-face $g$ in $\alpha$ such that either $g \in B d(B)$ or $g \notin B$. Let $f$ be the 2-face of $A$ that includes $g$.

Case 1: $g \in B d(B)$. Thus, $(f, g)$ is a free pair for both $A$ and $B$. Let $A^{\prime}=A \backslash\{f, g\}$ and $B^{\prime}=B \backslash\{f, g\}$. We have $C \leq B^{\prime} \leq A^{\prime}, A^{\prime} \searrow C$ (by Prop. 19) and $B^{\prime} \searrow C$ (also by Prop. 19], thus by the recurrence hypothesis $A^{\prime} \searrow B^{\prime}$. It can be seen that any sequence of collapse operations from $A^{\prime}$ to $B^{\prime}$ is also a sequence of collapse operations from $A$ to $B$.

Case 2: $g \notin B$. Thus, $(f, g)$ is a free pair for $A$ that is not in $B$, let $A^{\prime}=A \backslash\{f, g\}$. We have $C \leq B \leq A^{\prime}, A^{\prime} \searrow C$ (by Prop. 19) and $B \searrow C$, thus by the recurrence hypothesis $A^{\prime} \searrow B$ hence $A \searrow B$.

The following theorem summarises Props. 19 and 20 .

Theorem 21 (Confluences). Let $M \subseteq \mathbb{F}_{2}^{n}$ be a pseudomanifold, and let $C \leq B \leq A \leq M$ be such that A collapses onto $C$. Then, $A$ collapses onto $B$ if and only if $B$ collapses onto $C$.

\section{Minimal Simple Sets in Pseudomanifolds}

Informally, a minimal simple set [11]13] is a simple set which does not strictly include any other simple set. In this section, we first establish the equivalence between the notions of simple cell and minimal simple set in pseudomanifolds (Prop. 25). Then we demonstrate that, in such spaces, any simple set can be fully detached, while preserving topology, by iterative detachment of simple cells, in any possible order (Th.26).

Definition 22 ([13]). Let $X \leq \mathbb{F}^{n}$ and $S \sqsubseteq X$. The subcomplex $S$ is a minimal simple set (for $X$ ) if $S$ is a simple set for $X$ and $S$ is minimal with respect to the relation $\sqsubseteq$ (i.e. $Z=S$ whenever $Z \sqsubseteq S$ and $Z$ is a simple set for $X)$.

Proposition 23 ([12]). Let $M \subseteq \mathbb{F}_{2}^{n}$ be a pseudomanifold, and let $S \sqsubseteq X \preceq M$ such that $S$ is a minimal simple set for $X$. Then, $S$ is connected. 
This property of minimal simple sets indeed holds in more general conditions, see [13].

Proposition 24 (proved in [12]). Let $M \subseteq \mathbb{F}_{2}^{n}$ be a pseudomanifold, let $X \leq M$ be a connected 2-complex, let $S \sqsubseteq X$ be a simple subcomplex of $X$, and let $f$ be a facet of $S$ such that $\operatorname{Att}(\hat{f}, X)$ is not empty and not connected. Then, $X \otimes \hat{f}$ is an extension of $\operatorname{Att}(\hat{f}, X)$.

Proposition 25. Let $M \subseteq \mathbb{F}_{2}^{n}$ be a pseudomanifold, and let $S \sqsubseteq X \preceq M$ such that $S$ is a minimal simple set for $X$. Then, $S$ is necessarily a 1-cell or a 2-cell.

Proof. Suppose that $S$ is not reduced to one cell. Then, each facet of $S$ must be nonsimple for $X$. No facet $f$ of $S$ is such that $\operatorname{Att}(\hat{f}, X)=\emptyset$. If $S$ contains a 1-facet, then let $f$ be such a facet. If $S$ is a pure 2-complex, then at least one 2-face of $S$ must include a free face for $X$, otherwise $X$ could not collapse onto $X \otimes S$, let us assume that $f$ is such a 2-face. Let $A=\operatorname{Att}(\hat{f}, X)$. In both cases $(\operatorname{dim}(f)=1$ or $\operatorname{dim}(f)=2)$, we know that $A$ is disconnected. From now, we suppose that $\operatorname{dim}(f)=2$ (the case where $\operatorname{dim}(f)=1$ is similar and simpler).

From Prop. 23, $S$ is connected and from Props. 9 and 2, $\operatorname{Att}(S, X)$ is connected. Without loss of generality, we assume that $X$ is connected (otherwise we replace $X$ by the component of $X$ that includes $S$ ). By Prop. 24, each component of $X \otimes \hat{f}$ includes exactly one component of $A$. Let $X_{1}$ be the component of $X \otimes \hat{f}$ that includes $\operatorname{Att}(S, X)$ (and thus also $X \otimes S$ ), and let $A_{1}$ be the component of $A$ that is in $X_{1}$. Let $g$ and $h$ be the two 1-faces of $\hat{f}^{*} \backslash A$ that include each a 0-face of $A_{1}$. Obviously $(f, g)$ is a free pair for $X$, let $X^{\prime}=X \backslash\{f, g\}$. Remark that $h$ is a facet of $X^{\prime}$. We have $X \searrow X^{\prime}$ and $X \searrow X \otimes S$, by Prop. 19 we deduce $X^{\prime} \searrow X \otimes S$.

Let $J=\left\langle\left(f_{i}, g_{i}\right)\right\rangle_{i=1}^{\ell}$ be a collapse sequence from $X^{\prime}$ to $X \otimes S$. Let $t \in[1, \ell]$ be such that $f_{t}=h$. It can be seen that $g_{t} \notin X_{1}$ (otherwise the result of the collapse operation would be disconnected, for by construction any path in $X^{\prime}$ from $X \otimes S$ to the remaining face in $h$ would contain $h$ ), and of course $f_{t} \notin X_{1}$. Furthermore, any other pair of $J$ is either in $X_{1}$ or in $X^{\prime} \backslash X_{1}$, since the only facet of $X^{\prime} \backslash X_{1}$ that includes a face of $X_{1}$ is $f_{t}$. Thus by Prop. $4 X^{\prime} \searrow X_{1}$, hence $X \searrow X_{1}$, i.e., $X \otimes X_{1}$ is a simple set for $X$. Remark that by construction, we have $X \odot X_{1} \sqsubseteq S$. Thus, the minimality of $S$ implies that $S=X \otimes X_{1}$, hence $\operatorname{Att}(S, X)=A_{1}$.

It is plain that $\hat{f} \searrow A_{1}$, thus by Prop. [6 we have $X_{1} \cup \hat{f} \searrow X_{1}$; and since $X \searrow X_{1}$, by Prop. 20 we deduce that $X \searrow X_{1} \cup \hat{f}$, i.e., $X \otimes\left(X_{1} \cup \hat{f}\right)$ is a simple set for $X$. This contradicts the minimality of $S$, since $X \otimes\left(X_{1} \cup \hat{f}\right) \sqsubseteq S$.

From Props. 25 and 19, we derive straightforwardly our main theorem.

Theorem 26. Let $M \subseteq \mathbb{F}_{2}^{n}$ be a pseudomanifold, and let $S \sqsubseteq X \leq M$ such that $S$ is a simple set for $X$. Then:

(i) there is a facet of $X$ in $S$ which is simple for $X$; and

(ii) for any cell $\hat{f}$ in $S$ which is simple for $X, S \otimes \hat{f}$ is a simple set for $X \otimes \hat{f}$.

\section{Conclusion}

In this article we have established, in the case of digital 2-D pseudomanifolds, a confluence property of the collapse operation (Th.21). From this result, we have proved that 
in pseudomanifolds, any minimal simple set is a simple cell (Prop. 25). This led us to the property stating that any simple set can be removed by iterative removal of simple cells in any order (Th.26).

It is indeed possible to retrieve Ronse's theorem (Th. 1) from the results presented above, based on the equivalence between $\mathbb{Z}^{2}$ equipped with a $(8,4)$-adjacency framework and the set of pure 2-complexes in $\mathbb{F}^{2}$ [7]. To this aim, it is necessary to prove that any subcomplex $S \sqsubseteq X$ (where $X$ is a pure 2-complex in $\mathbb{F}^{2}$ ) that is strongly deletable for $X$, is also simple for $X$ in the sense of Def. 8 (the converse also holds). The Jordan's theorem is needed for this proof, which is not in the scope of the present article.

\section{References}

1. Bertrand, G.: On topological watersheds. Journal of Mathematical Imaging and Vision 22 (2-3), 217-230 (2005)

2. Bertrand, G.: On critical kernels. Comptes Rendus de l'Académie des Sciences, Série Mathématiques 1(345), 363-367 (2007)

3. Bing, R.H.: Some aspects of the topology of 3-manifolds related to the Poincaré conjecture. Lectures on Modern Mathematics II, pp. 93-128 (1964)

4. Davies, E.R., Plummer, A.P.N.: Thinning algorithms: A critique and a new methodology. Pattern Recognition 14(1-6), 53-63 (1981)

5. Giblin, P.: Graphs, surfaces and homology. Chapman and Hall, Boca Raton (1981)

6. Yung Kong, T.: On topology preservation in 2-D and 3-D thinning. International Journal of Pattern Recognition and Artificial Intelligence 9(5), 813-844 (1995)

7. Yung Kong, T.: Topology-preserving deletion of 1's from 2-, 3- and 4-dimensional binary images. In: Ahronovitz, E. (ed.) DGCI 1997. LNCS, vol. 1347, pp. 3-18. Springer, Heidelberg (1997)

8. Kong, T.Y., Rosenfeld, A.: Digital topology: Introduction and survey. Computer Vision, Graphics, and Image Processing 48(3), 357-393 (1989)

9. Kovalesky, V.A.: Finite topology as applied to image analysis. Computer Vision, Graphics, and Image Processing 46(2), 141-161 (1989)

10. Maunder, C.R.F.: Algebraic topology. Dover, New York (1996)

11. Passat, N., Couprie, M., Bertrand, G.: Minimal simple pairs in the 3-D cubic grid. Journal of Mathematical Imaging and Vision 32(3), 239-249 (2008)

12. Passat, N., Couprie, M., Mazo, L., Bertrand, G.: Topological properties of thinning in 2-D pseudomanifolds. Technical Report IGM2008-5, Université de Marne-la-Vallée (2008)

13. Passat, N., Mazo, L.: An introduction to simple sets. Technical Report LSIIT-2008-1, Université de Strasbourg (2008)

14. Ronse, C.: A topological characterization of thinning. Theoretical Computer Science 43(1), 31-41 (1986)

15. Rosenfeld, A.: Connectivity in digital pictures. Journal of the Association for Computer Machinery 17(1), 146-160 (1970)

16. Zeeman, E.C.: Seminar on Combinatorial Topology. In: IHES (1963) 